\title{
Emergency management of ureteral stones: Evaluation of two different approaches with an emphasis on patients' life quality
}

\author{
Kemal Sarica $^{1}$, Bilal Eryildirim ${ }^{1}$, Cahit Sahin ${ }^{1}$, Özlem Kolçak Türkoğlu ${ }^{1}$, Murat Tuncer ${ }^{1}$, \\ Alper Coskun ${ }^{1}$, Hakan Akdere ${ }^{2}$ \\ ${ }^{1}$ Dr. Lütfi Kirdar Training and Research Hospital Urology Clinic, Istanbul, Turkey; \\ ${ }^{2}$ Trakya University, Faculty of Medicine, Urology Clinic, Edirne, Turkey.
}

\begin{abstract}
Summary Objectives: To evaluate the emergency management of obstructing ureteral calculi with two different techniques (SWL and URS) with an emphasis on patients life quality.

Methods: A total of 80 patients presenting with acute colic pain due to a single obstructing ureteral stone were treated within 24 hours following the onset of pain with two different approaches in a randomized manner. Patients requiring DJ stent placement and/or auxiliary measures after both procedures were excluded and the remaining 65 patients were evaluated [Group1: ESWL $(n=34)$; Group 2: URS $(n=31)]$. Patients were followed during 4-weeks period with respect to the analgesic requirement, number of renal colic attacks and emergency department visits along with the HRQOL scores.

Results: While 26 patients treated with URS (83.9\%) were stone-free, 24 cases in SWL were stone-free (70.6\%) after 4 weeks. Evaluation of the cases during this follow-up period demonstrated that cases undergoing SWL required signifcantly higher amount of analgesics when compared with URS group ( $p<0.001)$. In addition to the lower mean number of renal colic attacks and emergency department visits in URS group; both the mean HRQOL in terms of EQ-5D index and mean EQ-5D VAS values were also significantly higher in these cases when compared with the cases tretaed with SWL.

Conclusions: Due to the negative impact of stone related events after emergency SWL on patients HRQOL, emergency URS may be applied more effectively with the advantages of prompt fragmentation of the calculi along with the immediate relief of obstruction and pain.
\end{abstract}

KEY WORDS: Emergency treatment; Ureteral stone; Ureteroscopy; SWL; HRQOL.

Submitted 6 July 2016; Accepted 19 July 2016

\section{INTRODUCTION}

As a worldwide common pathology, urolithiasis affects about 5 to $10 \%$ of the general population (1). Despite the asymptomatic clinical course in a certain percent of the cases; ureteral calculi may cause obstruction and colic pain requiring an immediate management. Stone removal is often needed for relatively larger stones to remove the obstruction and colic related distressing symptoms necessitating analgesic use and emergency department (ED) visits which could have significant effects on the healthrelated quality of life (HRQOL) (2-8).

Placement of an ureteral stent or percutaneous nephrostomy tube are the alternatives when conservative medical management does not resolve symptoms (3-5). Currently shock wave lithotripsy (SWL) and ureterorenoscopy (URS) are commonly performed procedures and while EAU/AUA treatment guidelines accepted both approaches as preferred options (4). Stone free (SF) rates after SWL is probably lower, especially for mid and distal ureteral stones $(4,6)$.

Although both methods were performed successfully in an elective manner; accumulated data so far in demonstrated that emergency disintegration with SWL (management of the obstructing stones within 24 hours after the first colic attack) and also emergency removal with URS (where SWL system is unavailable or has been unsuccessful) may also be effective alternatives $(3,4)$. Each approach has its own advantages and disadvatages and although emergency SWL has been performed with acceptable stone free rates $(9,10)$; uretersocopic lithotripsy has been found to be more effective in the quick and complete relief of acute obstruction and related pain (11-13).

HRQOL is an estimate of freedom from impairment, disability or handicap (14). The concept of HRQOL is multidimensional and includes psychosocial, physical and emotional status, as well as patient autonomy and is applicable to a wide variety of medical conditions (1517). This brings the issue into the agenda that irrespective of the stone related factors, urologists should not solely focus on the stone free rates obtained but also on the possible effects of the procedure induced psychological and social life of the patients $(14,15)$. Related with this subject, although various studies focused on the stone free rates and stone related problems to some extent after treatment; to our knowledge no study so far has investigated the HRQOL of the patients after undergoing certain ureteral stone management procedures.

In this present prospective controlled study, in addition

No conflict of interest declared. 
to outline the efficacy of SWL and URS approaches performed in an emergent manner; we also aimed to evaluate the possible treatment related changes in the HRQOL of the patients undergoing these procedures.

\section{Patients AND MEthods}

Between October 2014 and May 2015, 80 adult patients (54 male and 26 female; M/F: 2.07) with acute colic pain due to a single obstructing opaque upper ureteral stone (5 to $10 \mathrm{~mm}$ ) were evaluated. Patients with multiple stones, previous stone surgery including stent placement and auxiliary procedures, congenital anomalies, active urinary tract infection, pregnancy or renal insufficiency were excluded.

Following acute pain management, emergency treatment of the stones within 24 hours after the onset of pain was performed. In addition to a detailed history and uro-genital examination, biochemical evaluation and urinalysis were performed. The study protocol has been approved by ethics committee of the institution. Although a noncontrast computed tomography (NCCT) was performed in all cases during colic attack; plain X-ray of the kidney, ureter and bladder (KUB), ultrasound and excretory urography were also done when necessary.

All cases were treated within 24 hours following the onset of pain with two different approaches (SWL and URS) in a randomized manner. Although medical expulsive therapy (MET)), observation or a planned elective therapy were offered; due to the distressing colic pain none has accepted these alternatives. Patients requiring DJ stent placement and/or auxiliary measures after both procedures were excluded due to the possible effects of these procedures on the HRQOL of the cases which may affect to interprete our final data. The remaining 65 patients were included in the study (Group 1: SWL ( $\mathrm{n}=$ 34); Group 2: URS $(n=31)$ ). Randomization was done by a simple method by generating a random digit (0-60 in each group). While even numbers were used for SWL, odd numbers used for URS. The advantages, disadvantages and possible complications of both procedures were explained and a written informed consent has been obtained.

SWL was performed with an electromagnetic lithotriptor (Compact Sigma, Dornier MedTech, Wessling, Germany) under analgesia. Semirigid ureteroscopy was performed with $8 \mathrm{Fr}$ ureteroscope (Karl Storz, Tuttlingen, Germany) under general anesthesia. In addition to the stone free and possible complication rates; patients were followed during 4weeks period (by accepting every week) with respect to the analgesic requirement (mg of diclofenac sodium applied), number of renal colic attacks and emergency department visits.

Lastly, as an important parameter the HRQOL of all cases were assessed by using EuroQol 5D (EQ-5D) scale ((comprising two different scales namely EQ5D index scale and the EQ-5D visual

Table 1.

Table 2. analogue scale (VAS)) which has been devaloped in 1987 by an international team, the European Quality of Life Group at the end of 4-weeks period (18).

Data are presented as mean \pm standard error of mean. By using Prism 5.0 (GraphPad Software, San Diego, CA), unpaired t test was used to evaluate the overall statistical significance between subgroups; $\mathrm{p}<0.05$ was considered to be significant.

\section{ResULTS}

A total of 65 patients were included and further evaluated (47 men and 18 women; M/F: 2.61), (Group 1: ESWL $(n=34) ;$ Group 2: URS $(n=31))$. The overall mean stone burden was $51.50 \pm 2.78 \mathrm{~mm}^{2}\left(30-96 \mathrm{~mm}^{2}\right)$. Patient as well as stone related characteristics in the whole group are being summarized in Table 1 .

Evaluation of our results after 4-weeks period revealed the following findings. Of all the 31 cases treated with URS: 26 cases (83.9\%) became completely stone free (SF), residual fragments (RF) were present in 5 cases (16.1\%). RF were removed by flexible URS. On the other hand, of the 34 cases undergoing SWL: 24 cases were completely SF (70.6\%), one case $(2.9 \%)$ had RF $(<4$ $\mathrm{mm}$ ), the procedure was unsuccessful in the remaining 9 cases (26.5\%) (Table 2). Ureteroscopic stone disintegration was performed in all the unsuccessful SWL treatments.

On the other hand, cases undergoing SWL required statistically significant higher amount of analgesics (mean value of analgesic requirement was $351.0 \pm 60.25 \mathrm{mg}(0$ $1200 \mathrm{mg})$ and $75.00 \pm 20.27 \mathrm{mg}(0-525 \mathrm{mg})$ respectively, $\mathrm{p}<0.001$ ). Additionally, the mean value of visual ana$\log$ scores during pain was also significantly higher in these cases $(p=0.0212)$. Same findings were true for the mean number of renal colic attacks and ED visits with significantly higher values in favour of SWL $(<0.001$ and 0.0097 respectively) (Table 3).

Regarding the possible negative impact of above mentioned parameters on the HRQOL of the cases after 4 weeks; the life quality scores in both groups were well evaluated (by using the mean index values of EQ-5D and

Evaluation of patient and stone characteristics in both groups.

\begin{tabular}{|lcccc|}
\hline & $\begin{array}{c}\text { Overall } \\
\mathbf{n = 6 5}\end{array}$ & $\begin{array}{c}\text { ESWL } \\
\mathbf{n = 3 4}\end{array}$ & $\begin{array}{c}\text { URS } \\
\mathbf{n = 3 1}\end{array}$ & $\mathbf{p}$ \\
\hline Age (year) & $40.50 \pm 1.73$ & $38.73 \pm 2.48$ & $42.27 \pm 2.41$ & 0.3109 \\
\hline Stone burden $\left(\mathrm{mm}^{2}\right.$ ) & $51.50 \pm 2.78$ & $47.40 \pm 2.84$ & $55.60 \pm 4.71$ & 0.1416 \\
\hline HU (hounsfield unit) & $764.4 \pm 37.59$ & $707.5 \pm 46.72$ & $821.3 \pm 57.82$ & 0.1312 \\
\hline Degree of hydronephrosis (grade) & $1.71 \pm 0,10$ & $1.53 \pm 0.14$ & $1.90 \pm 0.13$ & 0.0773 \\
\hline
\end{tabular}

Stone passage rates and required interventions after 4-weeks in both groups.

\begin{tabular}{|lcccc|}
\hline Stone free & 24 & 70.6 & 26 & 83.9 \\
\hline Residüel stone $\leq 4 \mathrm{~mm}$ & 1 & 2.9 & 0 & 0 \\
\hline Not stone free (requiring intervention) & 9 & 26.5 & 5 & 16.1 \\
\hline
\end{tabular}


Table 3.

Evaluation of the mean number of colic attacks, analgesic requirement, $E D$ visits and VAS in both groups during 4-weeks follow-up period.

\begin{tabular}{|lcccc|}
\hline $\begin{array}{c}\text { Overall } \\
\mathbf{n = 6 5}\end{array}$ & $\begin{array}{c}\text { ESWL } \\
\mathbf{n = 3 4}\end{array}$ & $\begin{array}{c}\text { URS } \\
\mathbf{n = 3 1}\end{array}$ & $\mathbf{p}$ \\
\hline No. of renal colic & $2.55 \pm 0.36$ & $4.30 \pm 0.54$ & $0.80 \pm 0.20$ & $<0.001$ \\
\hline No. of ED visit & $0.68 \pm 0.13$ & $1.03 \pm 0.23$ & $0.33 \pm 0.11$ & 0.0097 \\
\hline Analgesic required $(\mathrm{mg})$ & $213.3 \pm 36.30$ & $351.0 \pm 60.25$ & $75.00 \pm 20.27$ & $<0.001$ \\
\hline VAS during pain & $4.90 \pm 0.35$ & $5.70 \pm 0.38$ & $4.10 \pm 0.55$ & 0.0212 \\
\hline
\end{tabular}

Table 4.

Evaluation of the mean EQ-5D index values and EQ-5D VAS values in both groups, during 4-weeks follow-up period.

\begin{tabular}{|lcccc|}
\hline & $\begin{array}{c}\text { Overall } \\
\mathbf{n = 6 5}\end{array}$ & $\begin{array}{c}\text { ESWL } \\
\mathbf{n = 3 4}\end{array}$ & $\begin{array}{c}\text { URS } \\
\mathbf{n = 3 1}\end{array}$ & $\mathbf{p}$ \\
\hline Mean EQ-5D index & $0.82 \pm 0.13$ & $0.77 \pm 0.02$ & $0.87 \pm 0.01$ & 0.004 \\
\hline Mean EQ-5D VAS value & $78.92 \pm 1.35$ & $73.17 \pm 1.72$ & $84.67 \pm 1.49$ & $<0.001$ \\
\hline VAS during pain & $4.90 \pm 0.35$ & $5.70 \pm 0.38$ & $4.10 \pm 0.55$ & 0.0212 \\
\hline
\end{tabular}

safe, quick and more cost-effective manner with URS $(26,27)$. However; despite the advantage of an immediate decompression of the obstruction in one session, general anesthesia and hospitalization will be required in these cases $(4,11)$. Due to the prolonged obstruction along with the impact of repeated colic attacks requring analgesic medication and emergency department referral, emergency management of ureteral stones during or immediately after the first acute renal colic attack has been applied as a more reasonable alternative in selected cases $(3,10,28)$. Available limited data demostrated that both "SWL" and "Ureteroscopic lithotripsy" could be performed in an "emergent manner" to relieve the present obstruction and related colicky pain. However it is hard for the responsible endourologist to select and also offer one procedure as more advantageous than the other one depending solely on these similar outcomes.

mean values of EQ-5D VAS methods) after 4-weeks. Our results showed that cases undergoing SWL tended to have significantly lower scores indicating the negative effects of spontaneous passage of RF and additional procedures. Evaluation of the HRQOL scores in terms of EQ-5D instrument diemensions again demonstrated that the "pain/discomfort" and "anxiety/depression" dimensions were the most commonly and meaningfully affected dimensions (expressions as "some problems"). Our data revealed the mean indices of EQ-5D to be $0.77 \pm 0.02$ $(0.36-1.00)$ vs $0.87 \pm 0.01(0.76-1.00),(p=0.004)$ in cases undergoing SWL and URS respectively. Lastly, evaluation of mean EQ-5D VAS values demonstrated higher mean values in patients undergoing URS $(p<0.001)$ (Table 4). These findings indicated the meaningful adverse effects of SWL related events on the QOL of the cases treated for ureteral calculi. Comparative evaluation of our results with pooled European data of comparable age group did show similar mean index values for the dimensions evaluated (19).

\section{Discussion}

As a result of obstruction and colic pain requiring an emergency management in the majority of the cases, upper ureteral stones may significantly affect the life quality of the patients $(1,2)$. Currently both SWL and URS with different lithotriptor systems are acceptable alternatives in the management of these stones $(20,21)$. Although SWL seems to be the preferred method with its effective and safe nature; repeated treatments may prolong the total duration of the treatment during which fragment passage may cause obstruction, colic pain and urinary symptoms (22-25). In addition to distressing symptoms; severe obstruction will require a rapid stone removal in at least a certain subset of patients with relatively harder stones $(1,2,4,5)$.

On the other hand, clinical use of the smaller scopes has led the endourologists to remove the ureteral calculi in a
Lastly, such stones could be a serious health problem due to the obstruction induced colic pain and associated distressing symptoms with significant adverse effects on the HRQOL of the cases $(7,8)$.

Health related quality of life assessment is an increasingly important aspect of contemporary medical practice which can be measured by general and/or disease-specific instruments (14). The concept of HRQOL is multidimensional and includes psychosocial, physical and emotional status, as well as patient autonomy and is applicable to a wide variety of medical conditions (15-17). Among the instruments used so far; as a generic, HRQOL instrument to measure health outcomes; EQ-5D scale has been devaloped in 1987 by an international team, the European Quality of Life Group. The validity of the EQ-5D has been assessed within a number of different patient groups and within the general population in different countries. Currently there are 170 official language versions of EQ-5D (18). One of them is the Turkish version of EQ-5D, which was obtained from EuroQol (www.euroqol.org) and used in our study. This instrument comprises two different scales namely EQ-5D index scale and the EQ-5D visual analogue scale (VAS). The EQ-5D index scale currently comprises a questionnaire with five dimensions (mobility, self-care, usual activities, pain/discomfort and anxiety/depression). Each dimension of the EQ-5D is divided into three degrees of severity as "no problem", "some problems", or "major problems". A single index score can be produced using information from these five dimensions. The EQ-5D index score range from -0.59 to 1 and includes a worse than death measure (negative score), outside the range of 0 (dead) to 1 (perfect health). Second scale is the EQ-5D VAS scale and it is a $20-\mathrm{cm}$ visual analogue scale where the respondent is asked to mark his or her own current state of health on a thermometer-like line calibrated from 0 to 100 .

In light of the data given above, we may claim that emergency removal of ureteral calculi following pain manage- 
ment after first colic attack will further be advantageous by diminishing the negative effects of the stone induced symptoms on the HRQOL of the cases and this observation will further strengthen the judicious emergency application of both procedures in such cases. With this concept, we may realize that urologists should not solely focus on the SF rates obtained but also on the possible effects of the stone-induced distressing symptoms on the psychological and social life of the patients $(14,15)$. To our knowledge, no study so far has investigated HRQOL in patients with ureteral calculi undergoing SWL as well as URS in an emergent manner.

Regarding the possible changes in HRQOL in patients with stone disease Penniston and Nakada reported that these patients showed decreased HRQOL when compared to healthy adults. Using the SF-36 validated QOL questionnaire, they found that stone-bearing patients had lower scores on the general health and bodily pain domains, and female stone formers reported lower average QOL scores than male respondents (17). In 2009, again, Bensalah et al. reported multiple factors affecting HRQOL in such patients, including BMI, age and the number of surgical procedures performed (15). Lastly, Rabah et al. examined HRQOL of patients after lithotripsy procedure and stated that HRQOL of these cases was similar to the healthy controls (8).

In this present study, we aimed to evaluate the possible effects of two different ureteral stone management options (SWL vs URS) performed in an emergent manner on the HRQOL of the treated patients. Our results have clearly demonstrated that contrary to SWL application; ureteroscopic Ho-YAG disintegration of ureteral stones were found to be associated with higher SF rates after a single session with limited or no additional procedures which significantly lowered the mean number of both renal colics and ED visits along with the total amount of analgesic used. Relatively lower SF rates after a single session of SWL coupled with higher rates of additional procedures for RF removal may have a negative impact on patients life quality during this period. Evaluation of the data reported in the literature on this aspect so far did show that although $5-10 \%$ of the patients reported "extreme problems"; most of the patients had reported "no problem" for all domains. Our findings were in accordance with these data where most of the cases after SWL showed "some problems" related with two main dimensions ("pain/discomfort" and "anxiety/depression").

Thus our data indicate that in addition to the higher SF rates and limited additional procedures, emergency ureteroscopy may have certain advantages mentioned above which will definitely have positive effects on the HRQOL of treated cases. Our findings again seem to be further valuable by giving the chance for the practising urologists to offer this approach reliably based on the objective advantages of the procedure that will inevitably preferred by the patients.

Our current study has only one certain limitation; the number of the cases evaluated in this study might be small, but in light of the highly limited data available in the literature, we believe that our current findings will be contributive enough to a considerable extent. Furthermore to our knowledge this is the first report focusing on the life quality changes in cases undergoing two different stone treatment modalities (SWL vs URS).

\section{Conclusion}

Emergency ureteroscopic management of obstructive ureteral stones appears to be an effective treatment modality with comparable success as well as complication rates with SWL performed in the same manner. However, taking the statistically significant negative impact of stone related distressing events induced by the fragments after emergency SWL on the HRQOL of the treated cases, we believe that in skilled hands, emergent URS approach may be a better option than emergency SWL. We believe that further studies with larger series of patients including other indicative parameters are certainly needed.

\section{References}

1. Teichman JM. Clinical practice. Acute renal colic from ureteral calculus. N Engl J Med. 2004; 350:684-93.

2. Doublet JD, Tchala K, Tligui M, et al. In situ extracorporeal shock wave lithotripsy for acute colic due to obstructing ureteral stones. Scand J Urol Nephrol. 1997; 31:137-9.

3. Gettman MT, Segura JW. Management of ureteric stones: issues and controversies. BJU Int. 2005; 95:85-93.

4. Preminger GM, Tiselius HG, Assimos DG, et al. EAU/AUA: Nephrolithiasis Guideline panel. 2007 guideline for the management of ureteral calculi. J Urol. 2007; 178:2418-34.

5. Joshi HB, Obadeyi OO, Rao PN. A comparative analysis of nephrostomy, JJ stent and urgent in situ extracorporeal shock wave lithotripsy for obstructing ureteric stones. BJU Int. 1999; 84:264-9.

6. Bierkens AF, Hendrikx AJ, de la Rosette JJ, et al. Treatment of mid- and lower ureteric calculi: extracorporeal shock-wave lithotripsy vs laser ureteroscopy. A comparison of cost, morbidity and effectiveness. Br J Urol. 1998; 81:31-7.

7. Roehrborn CG. Acute relief or future prevention. is urology ready for preventive health care? Urology 2000; 56:12-9.

8. Rabah DM, Alomar M, Binsaleh S. Health related quality of life in ureteral stone patients: post-ureterolithiasis. Urol Res. 2011; 39:385-8.

9. Peschel R, Janetschek G, Bartsch G. Extracorporeal shock wave lithotripsy versus ureteroscopy for distal ureteral calculi: a prospective randomized study. J Urol. 1999; 162:1909-12.

10. Tiglui M, El Khadime MR, Tchala K, et al. Emergency extracorporeal lithotripsy (ESWL) for obstructing ureteral stones. Eur Urol. 2003; 43:552-5.

11. Jiang H, Wu Z, Ding Q. Ureteroscopy and holmium YAG laser lithotripsy as emergency treatment for acute renal failure caused by impacted ureteral calculi. Urology 2008; 72:504-7.

12. Conort P, Doré B, Saussine C. Comité Lithiase de l'Association Françaised'Urologie (Guidelines for the urological management of renal and ureteric stones in adults) ProgUrol. 2004; 14:1095-1102.

13. Kijvikai K, Haleblian GE, Preminger GM, et al. Shockwave lithotripsy or ureteroscopy for the management of proximal ureteral calculi: an old discussion revisited. J Urol. 2007; 178:1157-63. 
14. Last JM, Spasoff RA, Harris SS. A dictionary of Epidemiology. New York, Oxford University Press. 2001; Vol 4: p.148.

15. Bensalah K, Tuncel A, Gupta A, et al. Determinants of quality of life for patients with kidney stones. J Urol. 2008; 179:2238-43.

16. Alonso J, Ferrer M, Gandek B, et al. IQOLA Project Group. Health-related quality of life associated with chronic conditions in eight countries: results from the Internation. Qual Life Res. 2004; 13:283-98.

17. Penniston KL, Nakada SY. Health related quality of life differs between male and female stone formers. J Urol. 2007; 178:2435-40.

18. EuroQol Group (20-5-2008) EQ-5D available versions. http://www.euroqol.org/web/users/language_a.php.

19. Szende A, Williams A. Measuring Self-Reported Population Health: An International Perspective based on EQ-5D. SpringMed Publishing, (Budapest) Hungary; 2004.

20. Tiselius H.G. Removal of ureteral Stones with extracorporeal shock wave lithotripsy and ureteroscopic procedures. What can we learn from the literature in terms of results and treatment efforts. Urol Res. 2005; 33:185-90.

21. Stewart GD, Bariol SV, Moussa SA, et al. Matched pair analysis of ureteroscopy vs. shock wave lithotripsy for the treatment of upper ureteric calculi. Int J Clin Pract. 2007; 61:784-8.
22. Turna B, Akbay K, Ekren F, et al. Comparative study of extracorporeal shock wave lithotripsy outcomes for proximal and distal ureteric stones. Int Urol Nephrol. 2008; 40:23-9.

23. Ziaee SA, Halimiasl P, Aminsharifi A, et al. Management of 10$15 \mathrm{~mm}$ proximal ureteral stones: ureteroscopy or extracorporeal shockwave lithotripsy? Urology 2008; 71:28-31.

24. Karlsen SJ, Renkel J, Tahir AR, et al. Extracorporeal shockwave lithotripsy versus ureteroscopy for 5- to 10- $\mathrm{mm}$ stones in the proximal ureter: Prospective effectiveness patient-preference trial. J Endourol. 2007; 21:28-33.

25. Bagley DH. Expanding role of ureteroscopy and laser lithotripsy for treatment of proximal ureteral and intrarenal calculi. Curr Opin Urol. 2002; 12:277-80.

26. Lee YH, Tsai JY, Jiaan BP, et al. Prospective randomized trial comparing shock wave lithotripsy and ureteroscopic lithotripsy for management of large upper third ureteral stones. Urology 2006; $67: 480-4$.

27. Yencilek F, Erturhan S, Sarica K. Treatment of ureteral calculi with semi-rigid ureteroscopy: Where should we stop? Urol Int. 2010; 84:260-4.

28. Sarica K, Tanniverdi O, Aydın M, et al. Emergency ureteroscopic removal of ureteral calculi after first colic attack: is there any advantage? Urology 2011; 78:516-20.

\section{Correspondence}

Kemal Sarica, MD

saricakemal@gmail.com

Bilal Eryildirim, $M D$

bilaleryildirim@yahoo.com

Cahit Sahin, MD

cahitsahin129@gmail.com

Murat Tuncer, MD

murattuncer77@hotmail.com

Alper Coskun, MD

dr.alper05@gmail.com

Dr. Lütfi Kirdar Training and Research Hospital Urology Clinic

Tecerdagi Cad. Yakutlar Sitesi G/11 - Kartal/Istanbul, Turkey

Özlem Kolçak Türkoğlu, MD

ozlemkolcak@hotmail.com

Dr. Lütfi Kirdar Training and Research Hospital Radiology Clinic, Istanbul, Turkey

Hakan Akdere, $M D$

hakdere@yahoo.com

Trakya University, Faculty of Medicine, Urology Clinic, Edirne, Turkey 